\title{
The current state of biomarkers of mild traumatic brain injury
}

\author{
Han Jun Kim, ${ }^{1}$ Jack W. Tsao, ${ }^{1,2,3}$ and Ansley Grimes Stanfill ${ }^{4,5}$ \\ 'Department of Neurology, College of Medicine, University of Tennessee Health Science Center, Memphis, Tennessee, USA. \\ ${ }^{2}$ Department of Neurology, Memphis Veterans Affairs Medical Center, Memphis, Tennessee, USA. ${ }^{3}$ Children's Foundation

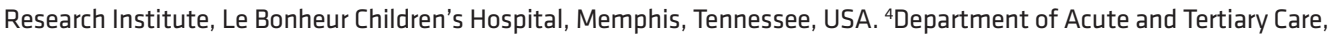 \\ College of Nursing, and 5 Department of Cenetics, Genomics, and Informatics, College of Medicine, University of Tennessee \\ Health Science Center, Memphis, Tennessee, USA.
}

Mild traumatic brain injury (mTBI) is a common occurrence, with over 3 million cases reported every year in the United States. While research into the underlying pathophysiology is ongoing, there is an urgent need for better clinical guidelines that allow more consistent diagnosis of mTBI and ensure safe return-to-play timelines for athletes, nonathletes, and military personnel. The development of a suite of biomarkers that indicate the pathogenicity of mTBI could lead to clinically useful tools for establishing both diagnosis and prognosis. Here, we review the current evidence for mTBI biomarkers derived from investigations of the multifactorial pathology of mTBI. While the current literature lacks the scope and size for clarification of these biomarkers' clinical utility, early studies have identified some promising candidates.

\section{Introduction}

Mild traumatic brain injury (mTBI), synonymous with concussion, is a prominent public health concern in the United States, with over 3 million injuries estimated annually (1). mTBI typically affects the frontal and temporal lobes of the brain, which are associated with executive function, learning, and memory (2). As these regions of the brain are still developing into the early twenties (3), student athletes are especially vulnerable to adverse effects following mTBI. Even more troubling, these repercussions can be exacerbated if a second concussive injury is sustained prior to the resolution of the first, leading to the phenomenon of second-impact syndrome (4). Each mTBI subsequent to the first causes greater cognitive decrement and a longer recovery time. While multiple studies have shown that cognition returns to preinjury levels, at least in the short term $(5,6)$, long-term neurodegenerative conditions, such as chronic traumatic encephalopathy (CTE), may develop $(7,8)$.

Despite concerns about the long-term effects of concurrent mTBIs, there are no clear clinical guidelines on when it is safe for a student athlete to return to play after mTBI. The current clinical standard only specifies that an injured athlete may return to play following symptom resolution $(9,10)$. Given that student athletes are typically highly motivated to return to the field, it is reasonable to suspect that athletic activities are likely to be resumed more quickly than they should and that young athletes might even minimize symptom severity. Furthermore, an athlete may appear asymptomatic using standardized concussion interview instruments; however, the biological mechanisms that underlie complete recovery appear to lag behind (11).

Here, we present the current state of research aimed at discovering biomarkers that relate to the effects of and recovery from concussion/mTBI. There is a critical need for the identification of biomarkers for mTBI to aid diagnosis and to inform the establishment of more effective clinical guidelines for treatment based on objective findings rather than subjective symptom complaints. The long-term goal of these studies should be to better design personalized therapy for concussed individuals and, especially, to address concerns about when it is safe for injured athletes to return to play as well as concerns of any individual who has sustained a concussion.

\section{Areas of discovery}

While the state of the science of mTBI biomarker research is still in its infancy, there are several promising areas of discovery related to the pathology of the injury. mTBI causes several unique pathologic changes in the brain, leaving it vulnerable to subsequent injury. Alterations include local pathophysiological changes, bloodbrain barrier (BBB) disruption, cerebral blood flow $(\mathrm{CBF})$ disturbance, axonal and neuronal cell body damage, 


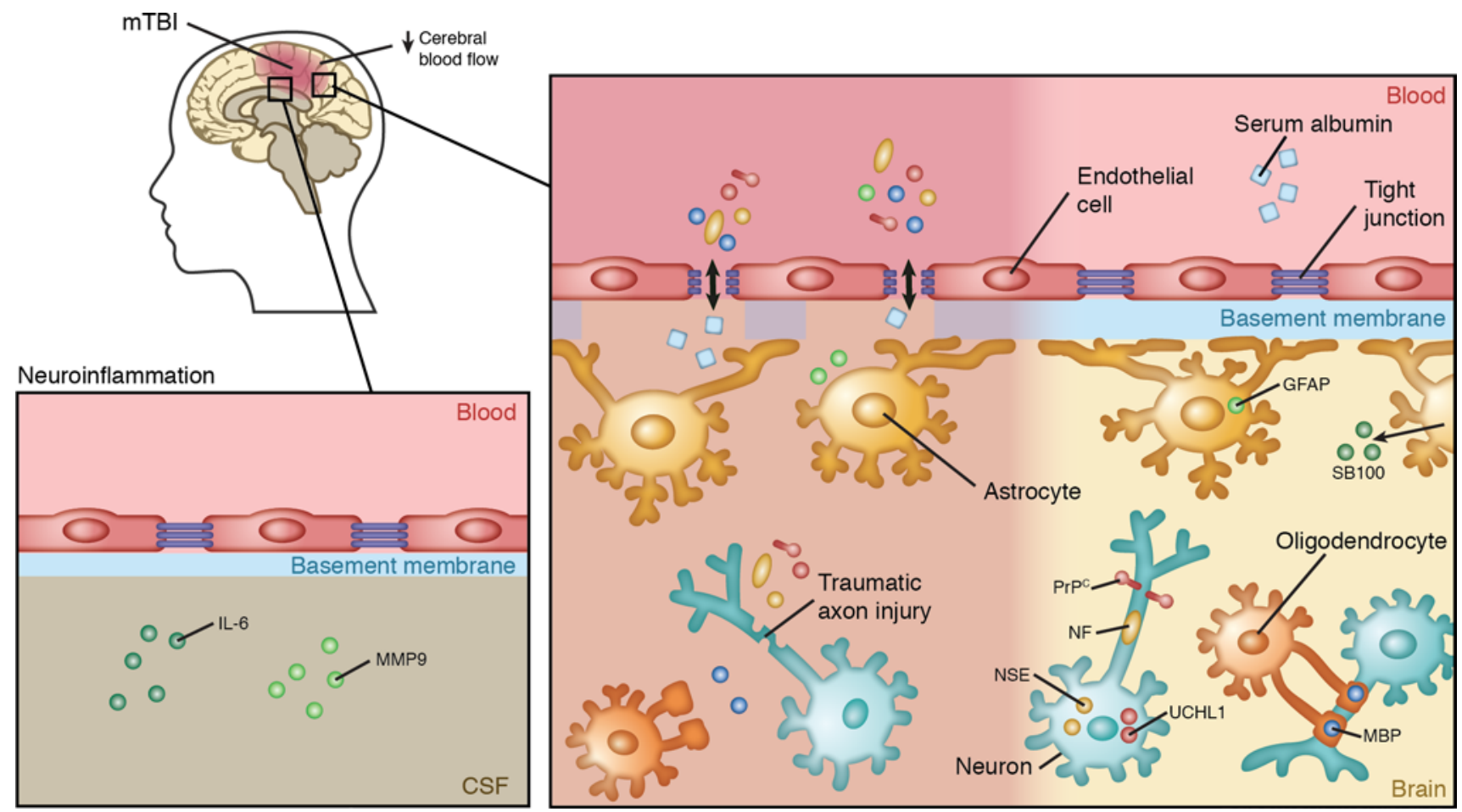

Figure 1. Physiological changes associated with mTBI that may facilitate biomarker discovery for this type of injury. Disruption of the blood-brain barrier (BBB) in response to mTBI may increase concentrations of brain-specific molecules in circulation, and evaluation of serum concentrations of these molecules may benefit mTBI diagnosis. Astrocyte-specific proteins, such as SB100 and GFAP, are found at high levels in the brain, and the presence of these in blood may indicate loss of BBB function or injury. Several neuron-specific proteins, including NSE, UCHL1, and PrPc, may be released from damaged neurons and could enter the blood if BBB integrity is compromised. Additionally, NFs can be released as the result of traumatic axonal injury. The presence of oligodendrocyte-specific proteins, such as MPB, in serum may also indicate mTBI. The presence of serum albumin, which is found at high levels in blood, in the CSF also indicates a breach of the BBB. Neuroinflammatory molecules, such as IL-6 and MMP9, in the CSF may also have potential to evaluate the extent of mTBI. Neuroimaging may also be useful, as CBF has been found to be decreased in some cases of mTBI.

and the neuroinflammatory response. All of these areas of pathophysiology are still under active investigation and show promise for potential mTBI biomarkers, as discussed below and as illustrated in Figure 1.

\section{Pathophysiology of mTBI}

Examination of the precise mechanisms of concussion remains an active area of research, though several possible routes of pathogenicity have been proposed. For example, indiscriminate neurotransmitter release and unchecked ionic fluxes occur as a result of biomechanical injury to the brain (12-14). Excess excitatory neurotransmitter binding leads to further neuronal depolarization with efflux of potassium and influx of calcium. These ionic imbalances force the sodium-potassium $\left(\mathrm{Na}^{+}-\mathrm{K}^{+}\right)$pump to work overtime in an attempt to restore the neuronal membrane potential. As the $\mathrm{Na}^{+}-\mathrm{K}^{+}$pump requires ATP, increased demand results in energy crisis, and this energy deficit puts the brain in a vulnerable position for subsequent injury $(15,16)$.

Following the period of accelerated glucose metabolism to correct ion imbalances, the brain enters a period of depressed metabolism. Calcium accumulation, when left unchecked, may impair mitochondrial oxidative metabolism and directly activate cell death. Such intra-axonal calcium flux has been shown to disrupt neurofilaments and microtubules, thereby impairing posttraumatic neuronal connectivity $(12,17)$. The summation of these changes is thought to underlie both the short- and long-term symptomatology seen in mTBI.

\section{BBB disruption}

$\mathrm{BBB}$ disruption has been linked to a variety of neurological disorders, and it is associated with more severe forms of TBI (18). In mTBI, BBB failure can either be pathogenically acute or become chronic without full resolution (19). After the primary injury, BBB disruption increases the permeability of brain vasculature and 
can expose CNS proteins to inflammatory cells, resulting in antigen unmasking and generation of CNS protein-targeting autoantibodies (20). Subsequent injuries could grant these circulating autoantibodies passage into the CNS compartment where they might disrupt microglia, astrocytes, and possibly neurons themselves (18). While the presence of CNS autoantibodies does not necessarily suggest autoimmune or neurological disease, the presence of such autoantibodies has been documented in several CNS disorders, such as epilepsy (21).

\section{Biomarkers of BBB dysfunction}

Unfortunately, there is not a reliable technique for directly and noninvasively measuring BBB integrity and function; therefore, we will discuss peripheral markers of BBB dysfunction, which are indicative of cerebrospinal (CSF) molecules in serum and/or serum components in the CNS.

$\mathrm{CSF} /$ serum albumin ratio. The CSF/serum albumin ratio has been used to examine $\mathrm{BBB}$ disruption in patients with severe TBI and remains the standard biomarker for BBB integrity (22-24). An increased $\mathrm{CSF}$ /serum albumin ratio has been reported in patients with severe TBI and indicates that albumin from the blood has passed into the CSF, indicative of BBB damage (25). While an elevated CSF/serum albumin ratio is commonly reported for severe TBI, such changes have not been seen in concussion $/ \mathrm{mTBI}$, suggesting that either the $\mathrm{BBB}$ remains largely intact or the damage is transient, such that the CSF/serum albumin ratio is not sensitive enough to detect mTBI-associated BBB damage (11). Results from Shahim et al. support this notion, as in their small cohort study, the CSF/serum albumin ratio was unaltered in 28 professional athletes with postconcussive symptoms (26). Despite the current lack of utility of this method for measuring possible BBB disruption in mTBI, more work should be done, perhaps with more sensitive techniques, such as mass spectrometry and/or proteomics.

Tight junction proteins. Tight junction proteins, such as the integral membrane protein occludin, have been suggested as another possible biomarker of mTBI-associated BBB dysfunction (27). It is likely that the levels of such proteins would be disrupted in BBB injury. Unfortunately, as occludin is not specific to the brain, its use as biomarker for diagnosis or confirmation of mTBI has limitations (28). Other families of brain-specific tight junction proteins may prove to be more selective biomarkers of BBB integrity, but research in this area is lacking.

$\mathrm{S100B}$. S100B is an astrocyte-specific CNS protein that is known to be upregulated in several neurological conditions, including ischemia (29). Under normal conditions, S100B is concentrated in CSF, but due to the leak that occurs upon BBB disruption, serum S100B levels increase (29). Though the exact functions of $\mathrm{S} 100 \mathrm{~B}$ remain elusive, sensitivity of $\mathrm{S100B}$ as a marker of BBB leakage is comparable to that of the CSF/ serum albumin ratio (18). It is possible that reactive glia upregulate S100B; however, a study by Marchi et al. refutes this possibility and shows that the rise in serum S100B levels is too early after injury to be due to synthesis by glial cells (29).

$\mathrm{S} 100 \mathrm{~B}$ is an important mTBI biomarker candidate, because serum levels of this protein have already been used experimentally to rule out mTBI in emergency room settings (30). In a clinical study of 1,560 patients with minor head injury, initial blood S100B levels had a high negative predictive value of 99.7\% (30). This result shows blood S100B levels have great potential as a screening tool for physicians who may not be inclined to perform head CT in certain cases, such as younger patients who might need to avoid radiation or pregnant women. Unfortunately, lack of specificity, and the presence of extracerebral sources of S100B in peripheral blood, limits the diagnostic value. Moreover, elevated serum S100B has also been detected in patients with extracranial pathology, such as traumas and burns (31). Further caution is warranted by another study that found no relationship between serum S100B concentration and mTBI severity in a sample of 94 patients (32).

Plasma-soluble prion protein. Cellular prion protein $\left(\mathrm{PrP}^{\mathrm{c}}\right)$ is a ubiquitous glycoprotein that is at its highest concentration in the CNS (33). Because $\mathrm{PrP}^{\mathrm{c}}$ is located entirely within the extracellular domain of the plasma membrane, it is reasonable to suspect that it may be released during a concussive event through $\mathrm{BBB}$ disruption (33). Indeed, transcription of the prion protein gene $(P R N P)$ is upregulated in a rat model of concussion following blast exposure, resulting in increased serum levels $(34,35)$.

Little work has been done to measure changes in plasma-soluble $\mathrm{PrP}^{\mathrm{c}}$ in humans. For example, in a small 6-person cohort of concussed Canadian collegiate athletes, plasma $\mathrm{PrP}^{\mathrm{c}}$ levels increased within 24 hours after mTBI compared with those in healthy young adults and nonconcussed athletes (33). $\operatorname{PrP}^{\mathrm{c}}$ was also increased in athletes examined during the off-season, a sign that $\operatorname{Pr}^{\mathrm{c}}$ may be persistently elevated following mTBI (33). Further studies in larger cohorts are needed to confirm and replicate these results; however, these studies point to $\operatorname{PrP}^{\mathrm{c}}$ as a potentially relevant biomarker for identifying concussed athletes. 


\section{CBF disturbance}

After mTBI, there is a decrease in cerebral autoregulatory capacity that disturbs CBF (36). This impairment in vasoreactivity after trauma has been attributed to increased endothelial NO production that in turn impairs smooth muscle response (37). Neuroimaging studies have confirmed that decreased CBF after mTBI is associated with neurocognitive deficits and symptom severity (38). For example, acute mTBI symptoms, such as migraines, have been associated with altered CBF to the trigemino-cortical pathway (39). Animal studies indicate that cognitive deficits and progressive decline in CBF can persist up to a year after the initial damage $(40,41)$.

\section{Biomarkers of CBF disturbance}

Neuroimaging. Current advanced neuroimaging techniques allow detection of neuropathological alterations in mTBI patients at the millimeter to submillimeter level (42). In a functional MRI study of concussed collegiate football players, decreased CBF was detected in several regions (1). While most individuals exhibited restored $\mathrm{CBF}$ at one month, a subset of individuals had a persistent decrease in regional CBF (1). Subsequent studies, have shown that at later time points this type of regional hypoperfusion in the frontal, prefrontal, and temporal cortices associates with neurocognitive deficits (1). These results suggest that determination of regional $\mathrm{CBF}$ detection via neuroimaging has potential as an objective biomarker for tracking symptoms and recovery after concussion. A study of 44 collegiate football athletes showed decreased $\mathrm{CBF}$ in the dorsal midinsular cortex (dmIC) and superior temporal sulcus, both of which are important for higher cognitive functioning, as compared with healthy controls one week after injury (43). Furthermore, CBF in dmIC at one-month after injury inversely correlated with concussion severity and symptom resolution (43). Future neuroimaging studies should examine not only functional activity after $\mathrm{mTBI}$ but also differences in functional connectivity as potential biomarker candidates (43).

\section{Traumatic axonal injury}

Multiple regions of the brain can suffer axonal injury after concussion due to sheering forces that place tension on axon fibers (44). While immediate axonal disconnection typically does not occur following mTBI, the aforementioned disturbance of the cellular environment caused by excess neurotransmitter release can result in secondary axotomy (45). Secondary axotomy occurs where the initial injury disturbs membrane homeostasis, which in turn causes axonal damage and threatens cell integrity (46). Such focal axonal (Wallerian) degeneration can lead to further damage through demyelination (47).

\section{Biomarkers of traumatic neuronal/axonal injury}

Tau protein. The axonal protein tau stabilizes microtubules, and tau-dependent regulation of microtubule dynamic stability allows cytoskeleton reorganization. Tau also influences axonal transport by its interactions with the dynein and kinesin motor proteins. Tau is subject to posttranslational modifications, including methylation and glycation; therefore, tau behavior is subject to change in response to injury (48) and is likely to vary from its normal physiological function in mTBI. For example, binding affinity of tau is decreased by posttranslation modification (1); thus, hyperphosphorylated tau ( $\mathrm{p}$-tau) destabilizes microtubules and alters axonal transport, leading to impaired neuronal function (1).

Animal models have shown a link between mTBI and an increase in p-tau depositions, which are associated with neurobehavioral abnormalities (1). While human studies have been limited, extensive p-tau pathology throughout the brain has been observed in late-stage CTE, which is considered a sequelae of multiple mTBIs (1). However, the presence of p-tau alone may not be CTE specific, as patients with temporal lobe epilepsy also exhibit similar patterns of tau deposition in resected tissue (49). Pathological tau deposition in the brain is common among familial neurodegenerative disorders, including Alzheimer disease; therefore, p-tau alone has limitation as a mTBI-specific marker. While p-tau may not be specific for mTBI, a recent study of 196 patients with acute TBI found that the ratio of p-tau to total tau served as a good diagnostic and prognostic marker for acute TBI across different severities, as measured by Glasgow Coma Scale (GCS) scores (50). This result suggests that tau still does have potential as a biomarker of mTBI, warranting further study.

UCHL1. The deubiquitinase ubiquitin carboxyl-terminal hydrolase isoenzyme L1 (UCHL1) is highly expressed in the neuronal cytoplasm $(51,52)$. While UCHL1 only comprises approximately $1 \%-2 \%$ of total soluble protein in the brain, it is also present in the peripheral nervous system at neuromuscular 
junctions $(53,54)$. Studies of UCHL1 as a serum biomarker have had conflicting findings. In a small study of nine mTBI participants, serum UCHL1 was elevated but was not proportional to white matter damage (55). A larger, prospective cohort study of 96 patients with mild $(n=86)$ to moderate $(n=10)$ TBI showed that UCHL1 was detectable in serum within 1 hour of injury and associated with measures of injury severity, including GCS score, lesions seen during brain imaging, and the need for neurosurgical intervention (56). In contrast, another study of 15 collegiate football players found no significant difference in serum levels of UCHL1 between mTBI-positive individuals and controls who did not sustain concussion (57). These mixed results warrant caution for future efforts into this biomarker.

Neurofilaments. Neurofilaments (NFs) are of interest as potential mTBI biomarkers and are specific to the CNS. As NFs are found in axons, which are extremely sensitive to concussion-induced damage, NF changes have potential to be an excellent biomarker of mTBI-triggered axonal damage (58-60). NFs have three components (light, medium, and heavy chains), the latter of which can be phosphorylated (pNFH). $\mathrm{pNFH}$ is responsible for protecting NFs from degeneration (59). Though promising, the potential of $\mathrm{pNFH}$ as a biomarker of mTBI and recovery has not been thoroughly explored. While a rat model of concussion injury showed no increase in serum pNFH, a single study in mTBI patients found a rise in serum $\mathrm{pNFH}$ levels on days one and three after injury, with high sensitivity and specificity at both time points $(>96 \%)$ (59). Given these promising results, pNFH should be further explored in future prospective, replication studies in larger study populations.

NSE. The glycolytic enzyme neuron-specific enolase (NSE) is enriched in neuronal cell bodies and thus is seemingly suitable as a candidate as a marker of neuronal damage. Initially identified in serum and CSF of head trauma and comatose patients, NSE levels in CSF are proportional to TBI severity and associated with increased mortality rates in cases of moderate or severe TBI (61-63). Studies on CSF NSE levels in patients with mTBI are lacking. Additionally, NSE is sensitive to hemolysis, a significant limitation for a potential serum biomarker. NSE is also present in erythrocytes and endocrine cells (64); therefore, in vitro lysis of erythrocytes from blood contamination in CSF samples raises NSE levels and provides a potential confounder (65).

GFAP. Unlike NSE, the intermediate filament protein glial fibrillary acidic protein (GFAP) is almost exclusively expressed in astrocytes, though it can be found in Leydig cells of human testis $(64,66)$. GFAP levels in CNS have been suggested to improve TBI outcome prediction models and may be able to serve as a marker of intracranial injury $(67,68)$. In fact, GFAP in CSF has been reported to have higher diagnostic accuracy than S100B (69) and was recently shown to have excellent accuracy in differentiating mTBI patients from controls (70). While the best candidate biomarker for mTBI remains to be determined, GFAP does have this clear advantage over S100B.

$M B P$. Myelin basic protein (MBP) is a component of oligodendrocytes and the second most abundant protein in the CNS. Berger et al. found that there was no difference in initial levels of serum MBP in a pediatric population with $\mathrm{mTBI}$ as compared with controls; however, there was a significant difference in peak MBP levels between patients and controls (71). Unfortunately, MBP levels do not increase promptly (48-72 hours after injury), making it unlikely that MBP could be useful as a screening tool. Once measured, however, the elevations in peak MBP levels persisted up to 2 weeks and were specific for future intracranial hemorrhage (ICH), a potentially serious complication that can be occur after TBI (71). A persistent elevation in MBP following $\mathrm{mTBI}$ is promising and suggests that serum MBP may be useful for screening pediatric populations for mTBI. This marker would be especially useful in children who may not be able to communicate an mTBI event or symptoms to their caregiver. Further studies of peak serum MBP levels in adult populations may yield fruitful results of MBP as an mTBI biomarker.

Spectrin breakdown products. The cytoskeletal protein $\alpha$ II-spectrin is present in axons and neuronal presynaptic terminals. During cellular injury it is cleaved to form spectrin breakdown products (SBDPs) (72). While studies in this area are limited, Siman et al. identified a subset of mTBI patients with elevated plasma N-terminal $\alpha$ II-spectrin fragment (SNTF), which is an SBDP cleaved by calpain (73). In these patients, the elevated plasma SNTF was $100 \%$ sensitive and $75 \%$ specific for predicting cognitive performance 3 months after injury (72). In a subsequent study of concussed hockey players $(n=28)$, elevations in serum SNTF returned to baseline as postconcussive symptoms resolved (74). In contrast, Berger et al. evaluated SBDP in a small $(n=11)$ pediatric population and found no statistical difference between mTBI and control patients (75). Thus, there appears to be some potential utility in SBDP levels as a biomarker for return to play (72) and as a predictive marker for persistent postconcussive symptoms. 
NGAL. Neutrophil gelatinase-associated lipocalin (NGAL) belongs to a family of lipocalin proteins that bind or transport lipid and other hydrophobic molecules (76). While NGAL expression is sparsely detected in brain and peripheral nerves (77), it is currently accepted as a biomarker of primary and secondary kidney injury. These studies have found NGAL to be elevated in both blood and urine samples of patients $(78,79)$. Moreover, NGAL is upregulated in the CNS of animal models of cerebral diseases, such as ICH (80). More recent animal studies have shown enhanced NGAL expression in the hippocampus after TBI, with peak elevation at day 1 (81). In a subsequent human trial, TBI patients $(n=54)$ exhibited increased of NGAL elevation in serum compared with controls, an observation that was especially pronounced in patients with severe TBI (82). There is a paucity of data of using NGAL as a potential mTBI biomarker, but NGAL merits further investigation.

\section{Neuroinflammatory response}

The culmination of metabolic disturbances and neuronal damage ultimately trigger an inflammatory response that is characterized by local microglia infiltration and accumulation of peripheral immune cells $(83,84)$. Local inflammatory cells scavenge cellular debris and create a barrier between the intact and damaged neuronal tissues (85). Production of local cytokines, proteases, and reactive free radical species is also stimulated by the microglia, further promoting the inflammatory cascade (86). Increased permeability of the BBB following mTBI also allows accumulation of peripheral immune cells (84). It is currently unknown whether or not these inflammatory responses are beneficial and promote neuron survival. Regardless, the clear presence of inflammation after mTBI hints that this immune cascade may contain potential biomarkers.

\section{Biomarkers of neuroinflammation}

Interleukins and other acute-phase inflammatory response proteins. Increased concentrations of inflammatory proteins, such as IL-6, IL-8, and IL-10, in the CSF following injury have been reported in many studies (87-93). It is unclear if this immune response is a result of local production of immune mediators or leakage of these proteins across a compromised BBB. Regardless, there appears to be an association between proinflammatory proteins and poor clinical outcome in mTBI $(90,94,95)$. A pilot study of 16 pediatric mTBI patients showed that increased concentrations of both IL-6 and MMP9 had a sensitivity of $81 \%$ and specificity of $94 \%$ for mTBI diagnosis (96). Further studies of inflammatory response molecules might yield other potential biomarkers.

Marinobufagenin. As mentioned previously, increased BBB permeability due to mTBI causes an elevation of proteins in compartments that were previously inaccessible. Such damage causes elevation of a marinobufagenin (MBG), a cardiotonic steroid substance that is also released in response to renal artery stenosis (RAS) in humans (97). MBG not only initiates inflammation but also maintains the inflammatory response; therefore, MBG levels in the circulation have been suggested as a potential candidate mTBI biomarker. In one of the first studies in this area, 110 Division I football players donated urine samples for analysis of MBG at baseline and again for up to 20 days after concussion (98). Urine MBG levels were highly elevated in concussed players, typically peaked between two and five days after injury, and correlated with neurocognitive symptoms (98). The persistence of MBG in the urine was mixed among concussed players, with some maintaining MBG at levels even higher than what was observed in initial two-to-five day window (98). Based on these results MBG warrants further investigation as a potential biomarker for $\mathrm{mTBI}$, especially in the context of other inflammatory consequences of this injury.

\section{Genetic biomarkers}

As we move forward into an age of precision medicine, many genetic markers are also being tested for applicability as mTBI biomarkers. Potential genetic variations that affect the pathophysiologic biomarkers considered above could potentially contribute to the susceptibility of an individual to injury and to the likelihood of developing secondary sequelae, such as postconcussion syndrome and CTE, after an injury has occurred. Here, we will very briefly discuss two of the leading candidates, located in the genes encoding apolipoprotein $\mathrm{E}(A P O E)$ and brain-derived neurotrophic factor (BDNF).

$A P O E$. $A P O E$ gene is a common target for studies on neurodegeneration, and the epsilon 4 allele has been shown to be one of the biggest risk factors for development of Alzheimer's disease (99). Recent inquiries into $A P O E$ have expanded into identifying associations between $A P O E$ mutations and the risk of developing mTBI; however, the results have been mixed results. A recent systematic review concluded that the 
presence of the $A P O E$ epsilon 4 allele was noncontributory to mTBI diagnosis (100). However, the presence of the epsilon 4 allele may contribute to the risk for long-term symptomatology after mTBI, especially in relationship to cognitive impairment (101) and fatigue (102), although the epsilon 4 allele was not associated with sleep disturbances (103). Furthermore, mTBI in early life may increase the risk of Alzheimer's disease in later life (99), making further inquiry into such associations justifiable.

$B D N F$. Like $A P O E, B D N F$ has been associated with various types of neurodegeneration. The $B D N F^{\mathrm{val} 66 \mathrm{Met}}$ allele was previously linked to a higher risk of experiencing mTBI (104) and is also being investigated for its association with the potential for memory deficits following mTBI. Indeed, evaluation of a small sample of war veterans with mTBI $(n=110)$ found that carriers of the minor allele of rs1157659 have less functional connectivity and atrophy in the hippocampus as compared with those that are homozygous for the WT allele (105), an association that would increase the likelihood of memory problems.

Although interesting, as with other areas of genetic inquiry, caution must be used. For each of these potential candidates, the effect sizes and sample sizes are quite small. Much larger replication samples are needed in order to correct for false discovery rates and spurious results. However, the falling costs of sequencing and the increasing availability of deep-sequencing methods make genetic biomarkers attractive candidates for future inquiry.

\section{Conclusion}

Unfortunately, the literature on mTBI biomarkers consistently suffers from a lack of confirmation and replication across studies. Only a handful of studies address each biomarker in a clinical context that is relevant to mTBI, which hampers clinical utility of the results. Additional studies with larger sample sizes are sorely needed for clearer insight. Differences in patient age, sex, and underlying physiology can have potential implications in pathology and, therefore, can either mask or amplify the importance of a particular biomarker for mTBI. Inquiry into potential biomarkers should be done iteratively from both preclinical and clinical models, with considerations to the relative importance of each potential biomarker within the context of other demographic and physiological changes, as is the case with the ratio of p-Tau to total Tau. Each potential biomarker should be carefully considered for its future clinical applicability, with an eye to designing screening tests for bedside, battlefield, and sideline use. It is possible that the best screening tests may be developed from a union of several different markers. More detailed investigations into the pathophysiological consequences related to current list of mTBI biomarker candidates may yield further insights and more useful mTBI biomarkers for clinical practice.

Address correspondence to: Ansley Stanfill, 920 Madison Ave. \#542, Memphis, Tennessee 38163, USA. Phone: 901.448.1176; Email: astanfi4@uthsc.edu.

1. Choe MC. The pathophysiology of concussion. Curr Pain Headache Rep. 2016;20(6):42.

2. Stuss DT. Traumatic brain injury: relation to executive dysfunction and the frontal lobes. Curr Opin Neurol. 2011;24(6):584-589.

3. Miller DJ, et al. Prolonged myelination in human neocortical evolution. Proc Natl Acad Sci USA. 2012;109(41):16480-16485.

4. McCrory P. Traumatic brain injury: revisiting the AAN guidelines on sport-related concussion. Nat Rev Neurol. 2013;9(7):361362.

5. Slobounov S, Slobounov E, Sebastianelli W, Cao C, Newell K. Differential rate of recovery in athletes after first and second concussion episodes. Neurosurgery. 2007;61(2):338-344.

6. Bleiberg J, et al. Duration of cognitive impairment after sports concussion. Neurosurgery. 2004;54(5):1073-1078.

7. Blennow K, et al. Traumatic brain injuries. Nat Rev Dis Primers. 2016;2:16084.

8. Goldstein LE, McKee AC. Shining (laser) light on traumatic brain injury blood biomarkers. JAMA Neurol. 2017;74(9):1045-1047.

9. McCrory P, et al. Consensus statement on concussion in sport--the 4th International Conference on Concussion in Sport held in Zurich, November 2012. PM R. 2013;5(4):255-279.

10. Buckley TA, Baugh CM, Meehan WP, DiFabio MS. Concussion management plan compliance: A study of NCAA power 5 conference schools. Orthop J Sports Med. 2017;5(4):2325967117702606.

11. Zetterberg H, Blennow K. Fluid biomarkers for mild traumatic brain injury and related conditions. Nat Rev Neurol. 2016;12(10):563-574.

12. Giza CC, Hovda DA. The new neurometabolic cascade of concussion. Neurosurgery. 2014;75 Suppl 4:S24-S33.

13. Rao VL, Başkaya MK, Doğan A, Rothstein JD, Dempsey RJ. Traumatic brain injury down-regulates glial glutamate transporter (GLT-1 and GLAST) proteins in rat brain. J Neurochem. 1998;70(5):2020-2027.

14. Yi JH, Hazell AS. Excitotoxic mechanisms and the role of astrocytic glutamate transporters in traumatic brain injury. Neurochem Int. 2006;48(5):394-403

15. Vagnozzi R, et al. Temporal window of metabolic brain vulnerability to concussion: a pilot $1 \mathrm{H}$-magnetic resonance spectroscop- 
ic study in concussed athletes--part III. Neurosurgery. 2008;62(6):1286-1295.

16. Vagnozzi R, et al. Assessment of metabolic brain damage and recovery following mild traumatic brain injury: a multicentre, proton magnetic resonance spectroscopic study in concussed patients. Brain. 2010;133(11):3232-3242.

17. Giza CC, Hovda DA. The neurometabolic cascade of concussion. J Athl Train. 2001;36(3):228-235.

18. Marchi N, et al. Consequences of repeated blood-brain barrier disruption in football players. PLoS ONE. 2013;8(3):e56805.

19. Shlosberg D, Benifla M, Kaufer D, Friedman A. Blood-brain barrier breakdown as a therapeutic target in traumatic brain injury Nat Rev Neurol. 2010;6(7):393-403.

20. Vincent A, Bien CG, Irani SR, Waters P. Autoantibodies associated with diseases of the CNS: new developments and future challenges. Lancet Neurol. 2011;10(8):759-772.

21. Vincent A, Lang B, Kleopa KA. Autoimmune channelopathies and related neurological disorders. Neuron. 2006;52(1):123-138.

22. Tibbling G, Link H, Ohman S. Principles of albumin and IgG analyses in neurological disorders. I. Establishment of reference values. Scand J Clin Lab Invest. 1977;37(5):385-390.

23. Bowman GL, Kaye JA, Quinn JF. Dyslipidemia and blood-brain barrier integrity in Alzheimer's disease. Curr Gerontol Geriatr Res. 2012;2012:184042.

24. Pisani V, et al. Increased blood-cerebrospinal fluid transfer of albumin in advanced Parkinson's disease. J Neuroinflammation. 2012;9:188.

25. Blennow K, Hampel H, Weiner M, Zetterberg H. Cerebrospinal fluid and plasma biomarkers in Alzheimer disease. Nat Rev Neurol. 2010;6(3):131-144.

26. Shahim $\mathrm{P}$, et al. Astroglial activation and altered amyloid metabolism in human repetitive concussion. Neurology. 2017;88(15):1400-1407.

27. Cummins PM. Occludin: one protein, many forms. Mol Cell Biol. 2012;32(2):242-250.

28. Saitou M, et al. Mammalian occludin in epithelial cells: its expression and subcellular distribution. Eur J Cell Biol. 1997;73(3):222-231.

29. Marchi N, et al. Peripheral markers of brain damage and blood-brain barrier dysfunction. Restor Neurol Neurosci. 2003;21(34):109-121.

30. Zongo D, et al. S100-B protein as a screening tool for the early assessment of minor head injury. Ann Emerg Med. 2012;59(3):209-218.

31. Neher MD, Keene CN, Rich MC, Moore HB, Stahel PF. Serum biomarkers for traumatic brain injury. South Med J. 2014;107(4):248-255.

32. Metting Z, Wilczak N, Rodiger LA, Schaaf JM, van der Naalt J. GFAP and S100B in the acute phase of mild traumatic brain injury. Neurology. 2012;78(18):1428-1433.

33. Pham N, Akonasu H, Shishkin R, Taghibiglou C. Plasma soluble prion protein, a potential biomarker for sport-related concussions: a pilot study. PLoS One. 2015;10(2):e0117286.

34. Kochanek PM, et al. Screening of biochemical and molecular mechanisms of secondary injury and repair in the brain after experimental blast-induced traumatic brain injury in rats. J Neurotrauma. 2013;30(11):920-937.

35. Pham N, Sawyer TW, Wang Y, Jazii FR, Vair C, Taghibiglou C. Primary blast-induced traumatic brain injury in rats leads to increased prion protein in plasma: a potential biomarker for blast-induced traumatic brain injury. J Neurotrauma. 2015;32(1):58-65.

36. Jünger EC, et al. Cerebral autoregulation following minor head injury. J Neurosurg. 1997;86(3):425-432.

37. DeWitt DS, Prough DS. Traumatic cerebral vascular injury: the effects of concussive brain injury on the cerebral vasculature. J Neurotrauma. 2003;20(9):795-825.

38. Bonne O, et al. Cerebral blood flow in chronic symptomatic mild traumatic brain injury. Psychiatry Res. 2003;124(3):141-152.

39. Hodkinson DJ, et al. Primary somatosensory cortices contain altered patterns of regional cerebral blood flow in the interictal phase of migraine. PLoS One. 2015;10(9):e0137971.

40. Kochanek PM, Hendrich KS, Dixon CE, Schiding JK, Williams DS, Ho C. Cerebral blood flow at one year after controlled cortical impact in rats: assessment by magnetic resonance imaging. J Neurotrauma. 2002;19(9):1029-1037.

41. Xiong Y, Mahmood A, Chopp M. Animal models of traumatic brain injury. Nat Rev Neurosci. 2013;14(2):128-142.

42. Bigler ED. Neuroimaging biomarkers in mild traumatic brain injury (mTBI). Neuropsychol Rev. 2013;23(3):169-209.

43. Meier TB, Bellgowan PS, Singh R, Kuplicki R, Polanski DW, Mayer AR. Recovery of cerebral blood flow following sports-related concussion. JAMA Neurol. 2015;72(5):530-538.

44. Smith DH, Hicks R, Povlishock JT. Therapy development for diffuse axonal injury. J Neurotrauma. 2013;30(5):307-323.

45. Büki A, Povlishock JT. All roads lead to disconnection?--Traumatic axonal injury revisited. Acta Neurochir (Wien). 2006;148(2):181-193.

46. Smith DH, Wolf JA, Lusardi TA, Lee VM, Meaney DF. High tolerance and delayed elastic response of cultured axons to dynamic stretch injury. J Neurosci. 1999;19(11):4263-4269.

47. Mierzwa AJ, Marion CM, Sullivan GM, McDaniel DP, Armstrong RC. Components of myelin damage and repair in the progression of white matter pathology after mild traumatic brain injury. J Neuropathol Exp Neurol. 2015;74(3):218-232.

48. Wang Y, Mandelkow E. Tau in physiology and pathology. Nat Rev Neurosci. 2016;17(1):5-21.

49. Puvenna V, et al. Is phosphorylated tau unique to chronic traumatic encephalopathy? Phosphorylated tau in epileptic brain and chronic traumatic encephalopathy. Brain Res. 2016;1630:225-240.

50. Rubenstein $\mathrm{R}$, et al. Comparing plasma phospho tau, total tau, and phospho tau-total tau ratio as acute and chronic traumatic brain injury biomarkers. JAMA Neurol. 2017;74(9):1063-1072.

51. Doran JF, Jackson P, Kynoch PA, Thompson RJ. Isolation of PGP 9.5, a new human neurone-specific protein detected by high-resolution two-dimensional electrophoresis. J Neurochem. 1983;40(6):1542-1547.

52. Wilkinson KD, Lee KM, Deshpande S, Duerksen-Hughes P, Boss JM, Pohl J. The neuron-specific protein PGP 9.5 is a ubiquitin carboxyl-terminal hydrolase. Science. 1989;246(4930):670-673.

53. Jeter CB, Hergenroeder GW, Hylin MJ, Redell JB, Moore AN, Dash PK. Biomarkers for the diagnosis and prognosis of mild traumatic brain injury/concussion. J Neurotrauma. 2013;30(8):657-670.

54. Chen F, Sugiura Y, Myers KG, Liu Y, Lin W. Ubiquitin carboxyl-terminal hydrolase L1 is required for maintaining the structure 
and function of the neuromuscular junction. Proc Natl Acad Sci USA. 2010;107(4):1636-1641.

55. Kou Z, et al. Combining biochemical and imaging markers to improve diagnosis and characterization of mild traumatic brain injury in the acute setting: results from a pilot study. PLOS ONE. 2013;8(11):e80296.

56. Papa L, et al. Serum levels of ubiquitin C-terminal hydrolase distinguish mild traumatic brain injury from trauma controls and are elevated in mild and moderate traumatic brain injury patients with intracranial lesions and neurosurgical intervention. J Trauma Acute Care Surg. 2012;72(5):1335-1344.

57. Puvenna V, et al. Significance of ubiquitin carboxy-terminal hydrolase L1 elevations in athletes after sub-concussive head hits. PLoS One. 2014;9(5):e96296.

58. Johnson VE, Stewart W, Smith DH. Axonal pathology in traumatic brain injury. Exp Neurol. 2013;246:35-43.

59. Gatson JW, Barillas J, Hynan LS, Diaz-Arrastia R, Wolf SE, Minei JP. Detection of neurofilament-H in serum as a diagnostic tool to predict injury severity in patients who have suffered mild traumatic brain injury. J Neurosurg. 2014;121(5):1232-1238

60. Neselius S, Zetterberg H, Blennow K, Marcusson J, Brisby H. Increased CSF levels of phosphorylated neurofilament heavy protein following bout in amateur boxers. PLoS ONE. 2013;8(11):e81249.

61. Böhmer AE, et al. Neuron-specific enolase, S100B, and glial fibrillary acidic protein levels as outcome predictors in patients with severe traumatic brain injury. Neurosurgery. 2011;68(6):1624-1630.

62. Chiaretti A, et al. NGF, DCX, and NSE upregulation correlates with severity and outcome of head trauma in children. Neurology. 2009;72(7):609-616.

63. Ross SA, Cunningham RT, Johnston CF, Rowlands BJ. Neuron-specific enolase as an aid to outcome prediction in head injury. Br J Neurosurg. 1996;10(5):471-476.

64. Olsson B, Zetterberg H, Hampel H, Blennow K. Biomarker-based dissection of neurodegenerative diseases. Prog Neurobiol. 2011;95(4):520-534

65. Ramont L, Thoannes H, Volondat A, Chastang F, Millet MC, Maquart FX. Effects of hemolysis and storage condition on neuron-specific enolase (NSE) in cerebrospinal fluid and serum: implications in clinical practice. Clin Chem Lab Med. 2005;43(11):1215-1217.

66. Davidoff MS, Middendorff R, Köfüncü E, Müller D, Jezek D, Holstein AF. Leydig cells of the human testis possess astrocyte and oligodendrocyte marker molecules. Acta Histochem. 2002;104(1):39-49.

67. Czeiter E, et al. Brain injury biomarkers may improve the predictive power of the Impact outcome calculator. J Neurotrauma. 2012;29(9):1770-1778

68. McMahon PJ, et al. Measurement of the glial fibrillary acidic protein and its breakdown products GFAP-BDP biomarker for the detection of traumatic brain injury compared to computed tomography and magnetic resonance imaging. JNeurotrauma. 2015;32(8):527-533.

69. Korfias S, et al. Slight and short-lasting increase of serum S-100B protein in extra-cranial trauma. Brain Inj. 2006;20(8):867-872

70. Bogoslovsky T, et al. Increases of plasma levels of glial fibrillary acidic protein, tau, and amyloid $\beta$ up to 90 days after traumatic brain injury. J Neurotrauma. 2017;34(1):66-73.

71. Berger RP, Adelson PD, Pierce MC, Dulani T, Cassidy LD, Kochanek PM. Serum neuron-specific enolase, S100B, and myelin basic protein concentrations after inflicted and noninflicted traumatic brain injury in children. J Neurosurg. 2005;103(1 Suppl):61-68.

72. Kulbe JR, Geddes JW. Current status of fluid biomarkers in mild traumatic brain injury. Exp Neurol. 2016;275 Pt 3:334-352.

73. Siman R, et al. Evidence that the blood biomarker SNTF predicts brain imaging changes and persistent cognitive dysfunction in mild TBI patients. Front Neurol. 2013;4:190

74. Siman R, Shahim P, Tegner Y, Blennow K, Zetterberg H, Smith DH. Serum SNTF increases in concussed professional ice hockey players and relates to the severity of postconcussion symptoms. J Neurotrauma. 2015;32(17):1294-1300.

75. Berger RP, Hayes RL, Richichi R, Beers SR, Wang KK. Serum concentrations of ubiquitin C-terminal hydrolase-L1 and $\alpha I I-s p e c t r i n$ breakdown product $145 \mathrm{kDa}$ correlate with outcome after pediatric TBI. J Neurotrauma. 2012;29(1):162-167.

76. Lee S, et al. Lipocalin-2 is an autocrine mediator of reactive astrocytosis. J Neurosci. 2009;29(1):234-249.

77. Kjeldsen L, Cowland JB, Borregaard N. Human neutrophil gelatinase-associated lipocalin and homologous proteins in rat and mouse. Biochim Biophys Acta. 2000;1482(1-2):272-283.

78. Mishra J, et al. Identification of neutrophil gelatinase-associated lipocalin as a novel early urinary biomarker for ischemic renal injury. J Am Soc Nephrol. 2003;14(10):2534-2543.

79. Mishra J, et al. Neutrophil gelatinase-associated lipocalin (NGAL) as a biomarker for acute renal injury after cardiac surgery. Lancet. 2005;365(9466):1231-1238.

80. Dong M, Xi G, Keep RF, Hua Y. Role of iron in brain lipocalin 2 upregulation after intracerebral hemorrhage in rats. Brain Res. 2013;1505:86-92.

81. Zhao J, Xi G, Wu G, Keep RF, Hua Y. Deferoxamine attenuated the upregulation of lipocalin-2 induced by traumatic brain injury in rats. Acta Neurochir Suppl. 2016;121:291-294

82. Zhao J, et al. Early expression of serum neutrophil gelatinase-associated lipocalin (NGAL) is associated with neurological severity immediately after traumatic brain injury. J Neurol Sci. 2016;368:392-398.

83. Loane DJ, Byrnes KR. Role of microglia in neurotrauma. Neurotherapeutics. 2010;7(4):366-377.

84. Habgood MD, et al. Changes in blood-brain barrier permeability to large and small molecules following traumatic brain injury in mice. Eur J Neurosci. 2007;25(1):231-238

85. Shitaka Y, et al. Repetitive closed-skull traumatic brain injury in mice causes persistent multifocal axonal injury and microglial reactivity. J Neuropathol Exp Neurol. 2011;70(7):551-567.

86. Khuman J, et al. Tumor necrosis factor alpha and Fas receptor contribute to cognitive deficits independent of cell death after concussive traumatic brain injury in mice. J Cereb Blood Flow Metab. 2011;31(2):778-789.

87. Csuka E, Morganti-Kossmann MC, Lenzlinger PM, Joller H, Trentz O, Kossmann T. IL-10 levels in cerebrospinal fluid and serum of patients with severe traumatic brain injury: relationship to IL-6, TNF-alpha, TGF-beta1 and blood-brain barrier function. J Neuroimmunol. 1999;101(2):211-221.

88. Kossmann T, et al. Intrathecal and serum interleukin- 6 and the acute-phase response in patients with severe traumatic brain 
injuries. Shock. 1995;4(5):311-317.

89. Semple BD, Bye N, Rancan M, Ziebell JM, Morganti-Kossmann MC. Role of CCL2 (MCP-1) in traumatic brain injury (TBI): evidence from severe TBI patients and CCL2-/- mice. J Cereb Blood Flow Metab. 2010;30(4):769-782.

90. Kirchhoff C, et al. Cerebrospinal IL-10 concentration is elevated in non-survivors as compared to survivors after severe traumatic brain injury. Eur J Med Res. 2008;13(10):464-468.

91. Goodman JC, Van M, Gopinath SP, Robertson CS. Pro-inflammatory and pro-apoptotic elements of the neuroinflammatory response are activated in traumatic brain injury. Acta Neurochir Suppl. 2008;102:437-439.

92. Buttram SD, et al. Multiplex assessment of cytokine and chemokine levels in cerebrospinal fluid following severe pediatric traumatic brain injury: effects of moderate hypothermia. J Neurotrauma. 2007;24(11):1707-1717.

93. Phillips DJ, et al. Activin a release into cerebrospinal fluid in a subset of patients with severe traumatic brain injury. $J$ Neurotrauma. 2006;23(9):1283-1294

94. Kumar RG, et al. Acute CSF interleukin-6 trajectories after TBI: associations with neuroinflammation, polytrauma, and outcome. Brain Behav Immun. 2015;45:253-262.

95. Csajbok LZ, Nylén K, Öst M, Sonander H, Nellgård B. In-hospital C-reactive protein predicts outcome after aneurysmal subarachnoid haemorrhage treated by endovascular coiling. Acta Anaesthesiol Scand. 2015;59(2):255-264.

96. Berger RP, Ta'asan S, Rand A, Lokshin A, Kochanek P. Multiplex assessment of serum biomarker concentrations in well-appearing children with inflicted traumatic brain injury. Pediatr Res. 2009;65(1):97-102.

97. Tian J, et al. Renal ischemia regulates marinobufagenin release in humans. Hypertension. 2010;56(5):914-919.

98. Oliver J, et al. Comparison of neurocognitive testing and the measurement of marinobufagenin in mild traumatic brain injury: A preliminary report. $J$ Exp Neurosci. 2015;9:67-72.

99. Liu CC, Liu CC, Kanekiyo T, Xu H, Bu G. Apolipoprotein E and Alzheimer disease: risk, mechanisms and therapy. Nat Rev Neurol. 2013;9(2):106-118

100. Lawrence DW, Comper P, Hutchison MG, Sharma B. The role of apolipoprotein E episilon ( $\varepsilon$ )-4 allele on outcome following traumatic brain injury: A systematic review. Brain Inj. 2015;29(9):1018-1031.

101. Yang ST, et al. Accumulation of amyloid in cognitive impairment after mild traumatic brain injury. J Neurol Sci. 2015;349(12):99-104.

102. Sundström A, Nilsson LG, Cruts M, Adolfsson R, Van Broeckhoven C, Nyberg L. Fatigue before and after mild traumatic brain injury: pre-post-injury comparisons in relation to Apolipoprotein E. Brain Inj. 2007;21(10):1049-1054.

103. Lee $\mathrm{HH}$, et al. The association of apolipoprotein e allele 4 polymorphism with the recovery of sleep disturbance after mild traumatic brain injury. Acta Neurol Taiwan. 2017;26(1):13-19.

104. Dretsch MN, et al. Brain-derived neurotropic factor polymorphisms, traumatic stress, mild traumatic brain injury, and combat exposure contribute to postdeployment traumatic stress. Brain Behav. 2016;6(1):e00392

105. Hayes JP, et al. BDNF genotype is associated with hippocampal volume in mild traumatic brain injury [published online ahead of print July 28, 2017]. Genes Brain Behav. https://doi.org/10.1111/gbb.12403. 